\title{
Signaling in the plant cytosol: cysteine or sulfide?
}

Cecilia Gotor $1{ }^{*}$

Email gotor@ibvf.csic.es

Ana M. Laureano-Marín 1

Inmaculada Moreno 1

Ángeles Aroca 1

Irene García 1

Luis C. Romero 1

1 Instituto de Bioquímica Vegetal y Fotosíntesis, Consejo Superior de Investigaciones Científicias, Universidad de Sevilla, Avenida Américo Vespucio, 49, 41092 Seville, Spain

\section{Abstract}

Cysteine (Cys) is the first organic compound containing reduced sulfur that is synthesized in the last stage of plant photosynthetic assimilation of sulfate. It is a very important metabolite not only because it is crucial for the structure, function and regulation of proteins but also because it is the precursor molecule of an enormous number of sulfur-containing metabolites essential for plant health and development. The biosynthesis of Cys is accomplished by the sequential reaction of serine acetyltransferase (SAT) and $O$-acetylserine(thiol)synthase (OASTL). In Arabidopsis thaliana, the analysis of specific mutants of members of the SAT and OASTL families has demonstrated that the cytosol is the compartment where the bulk of Cys synthesis takes place and that the cytosolic OASTL enzyme OAS-A1 is the responsible enzyme. Another member of the OASTL family is DES1, a novel L-cysteine desulfhydrase that catalyzes the desulfuration of Cys to produce sulfide, thus acting in a manner opposite to that of OAS-A1. Detailed studies of the oas-al and 
des 1 null mutants have revealed the involvement of the DES1 and OAS-A1 proteins in coordinate regulation of Cys homeostasis and the generation of sulfide in the cytosol for signaling purposes. Thus, the levels of Cys in the cytosol strongly affect plant responses to both abiotic and biotic stress conditions, while sulfide specifically generated from the degradation of Cys negatively regulates autophagy induced in different situations. In conclusion, modulation of the levels of Cys and sulfide is likely critical for plant performance.

AQ1

\section{Keywords}

Abiotic stress

Arabidopsis

Autophagy

Biotic stress

Sulfur metabolism

\section{Introduction}

Sulfur is a macronutrient essential for plant, that is present in nature in different oxidation states, being sulfate the most common oxidized form. Plants, microorganisms and fungi, differently from animals, are able to reduce sulfate to sulfide and incorporate the reduced sulfur into organic compound through an energy-dependent process, originating a large variety of essential biomolecules. Cysteine (Cys) is the first organic compound containing reduced sulfur synthesized by the plant photosynthetic assimilation of sulfate (Takahashi et al. 2011). Cys is a very important metabolite not only as a proteinogenic amino acid, but also by its function as a precursor of essential biomolecules. In proteins, the thiol group of Cys, susceptible of oxidation to render disulfide bridges, stabilizes the tertiary and quaternary protein structures and, consequently, determines protein function (Haag et al. 2012), and often located in enzyme active sites are essential for catalysis (Richau et al. 2012). Moreover, the reversible conversion between free thiols groups and disulfide bridges is the basis of protein redox regulation (Buchanan and Balmer 2005). An equally important aspect of Cys is that is the precursor molecule of an enormous number of sulfur-containing metabolites essential for the 
development of plant life. These metabolites include the other sulfur amino acid, methionine, vitamins such as biotin and thiamine, cofactors such as $S$-adenosylmethionine (SAM), coenzyme A, molybdenum cofactor (CoMo), and lipoic acid and iron-sulfur clusters that participate in the electron transport (Droux 2004; Wirtz and Droux 2005; Van Hoewyk et al. 2008 ). Special mention deserves the antioxidant glutathione (GSH), regarded major determinant of cellular redox homeostasis (Foyer and Noctor 2011; Noctor et al. 2012), which is based on the reactivity of its thiol group. Equally other functions of GSH in response to adverse environmental conditions, like resistance to metal stress through GSH-derived peptides (Mendoza-Cozatl et al. 2011; Rea 2012), xenobiotic detoxification by GSH-conjugation (Dixon et al. 2002), and plant defense to pathogens, together with many other cysteine-derived metabolites involved in pathogenic responses (Rausch and Wachter 2005). Other example is the hormone ethylene, involved in many aspects of plant life, which is synthesized in the cytosol from methionine (Bleecker and Kende 2000). Therefore, we conclude that Cys plays a central role in the primary and secondary metabolism of the plant.

The biosynthesis of cysteine is the last step of the photosynthetic sulfate assimilation pathway, which comprises four stages: transport, activation, reduction and incorporation (Fig. 1). Sulfate from the soil is transported through the root plasma membrane by a proton/sulfate co-transport, which subsequently is loaded into the xylem vessels and distribute it to the entire plant. The uptake of sulfate is strictly controlled and is the most regulated step in the pathway. In Arabidopsis thaliana, 12 genes have been identified and classified into four different groups, depending on their substrate affinity and function (Takahashi 2010). Sulfate can be stored into vacuoles or transported to shoot chloroplasts to proceed with the assimilatory pathway.

\section{Fig. 1}

The photosynthetic sulfate assimilation pathway. The four steps are indicated. APS: adenosine 5'-phosphosulfate, GSH/GSSH: reduced/oxidized glutathione, Fd: ferredoxin, SAT: serine acetyltransferase, OASTL: $O$-acetylserine(thiol)lyase 


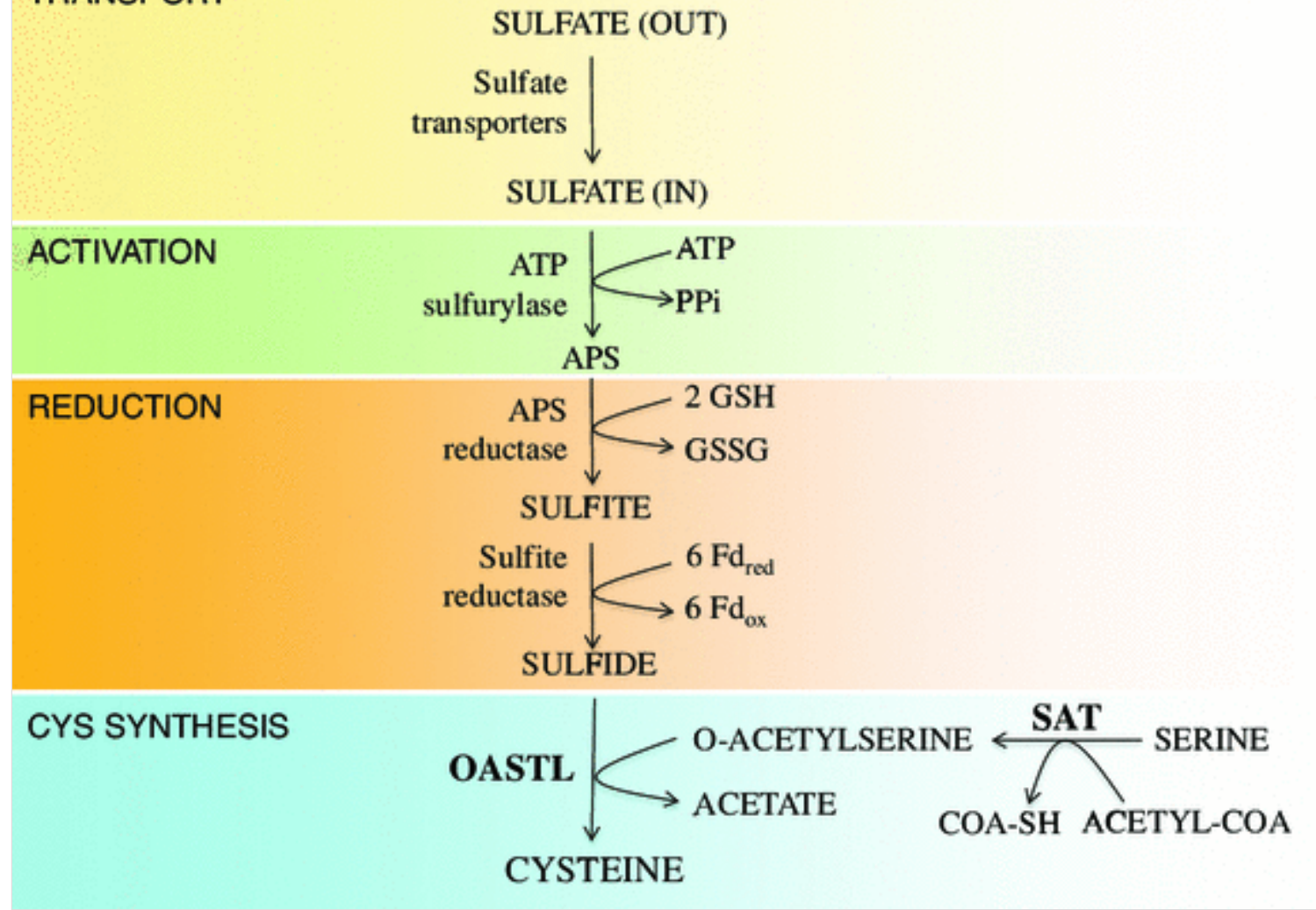

Before sulfate reduction, an activation step of sulfate must be produced to originate adenosine 5'-phosphosulfate (APS) with a redox potential lower than sulfate and consequently easily reduced by physiological electron donors (Fig. 1). This step is catalyzed by ATP sulfurylase, and in $A$. thaliana, three chloroplast and one cytosolic isoforms are presented (Hatzfeld et al. 2000a). APS is then reduced through two enzymatic steps located exclusively in chloroplasts, to produce sulfide, the most reduced inorganic form of sulfur (Fig. 1). In the firs step, APS is reduced to sulfite by APS reductase using GSH as reducing molecule, and in $A$. thaliana tree different chloroplast isoforms are described. The plant enzyme consists in two protein domains, the $\mathrm{N}$-terminal reductase and the $\mathrm{C}$-terminal thioredoxin/glutaredoxin-like domain (Setya et al. 1996; Kopriva and Koprivova 2004; Gutierrez-Marcos et al. 1996). APS reductions together with the sulfate uptake are primary steps of control of the sulfur assimilation, where the transcript levels are strictly regulated by sulfate availability (Yoshimoto et al. 2007; Buchner et al. 2004; Vauclare et al. 2002). In the second step of reduction, sulfite is reduced by sulfite reductase to sulfide in a six-electron reaction using reduced ferredoxin as reductant (Fig. 1). The enzyme is dependent on siroheme and $\mathrm{Fe}-\mathrm{S}$ centers as prosthetic groups. Differently from other enzymes of the pathway, are 
encoded by a low number of genes like in $A$. thaliana with only a single gene (Khan et al. 2010).

\section{Biosynthesis/metabolism of cysteine in Arabidopsis thaliana}

In the last stage of the photosynthetic sulfate assimilation pathway, the sulfide is incorporated into an amino acid skeleton to form cysteine, which represents the entry reaction of the most reduced inorganic sulfur compound into the metabolism in plants, but also in the human food chain. The biosynthesis of Cys takes place in the cytosol, plastids and mitochondria and is accomplished by the sequential reaction of the enzyme serine acetyltransferase (SAT) and the enzyme $O$-acetylserine(thiol)synthase (OASTL) (Fig. 1). SAT synthesizes the intermediary amino acid skeleton O-acetylserine (OAS) from actyl-CoA and serine. OASTL incorporates the sulfide to OAS to produce Cys, and requires pyridoxal-5'-phosphate (PLP) as cofactor. SAT and OASTL physically interact to form the hetero-oligomeric cysteine synthase complex, extensively studied in plants (Droux et al. 1998; Wirtz and Hell 2006, 2007; Francois et al. 2006 ). The SAT enzyme possesses a $\mathrm{N}$-terminal domain, rich in a $\alpha$-helix structures, involved in SAT/SAT interaction and a C-terminal domain responsible for the SAT/OASTL interaction and catalysis. Protein interactions within the complex strongly modify the kinetic properties of its components, enabling SAT to be active for the synthesis of OAS, but OASTL inactive and only the abundant free OASTL form synthesizes Cys. This complex is considered a regulatory circuit to modulate Cys production dependent on the intracellular sulfur state of the plant, as its formation is dependent on the relative amounts of sulfide and OAS (Yi et al. 2010; Jez and Dey 2013). Sulfide stabilizes the complex to the formation of OAS and the synthesis of Cys, but when OAS is in excess, the complex dissociates to reduce OAS production.

In the plant cells, a variety of different SAT and OASTL isoforms are present dependent on their location, thus resulting in different subcellular Cys pools. Besides, there is not agreement among the number of SATs and OASTLs in different photosynthetic organisms, generally with a higher proportion of OASTL proteins. These data induce to question about if all OASTL enzymes form complexes with their corresponding SAT enzymes, or on the contrary, some OASTLs have other functions not related with the 
primary Cys biosynthesis, as has been demonstrated in Arabidopsis (Romero et al. 2014). In the model organism Arabidopsis thaliana, there are five SAT enzymes (Howarth et al. 2003) and eight OASTL enzymes (Wirtz et al. 2004) (Fig. 2). Although the assimilatory reduction of sulfide takes place in the chloroplast, also the cytosol and the mitochondrion contain SAT and OASTL enzymes for the biosynthesis of cysteine. The three most abundant SATs are located in each compartment:

SAT1/SERAT2;1 (At1g55920) in the chloroplast; SAT3/SERAT2;2 (At3g13110) in the mitochondrion; and SAT5/SERAT1;1 (At5g56760) in the cytosol, and all three SATs interact with OASTLs to form the complex. Two other minor SAT isoforms: SAT2/SERAT3;1 (At2g17640) and SAT4/SERAT3;2 (At4g35640) are located in the cytosol and are suggested not to be able for interaction (Kawashima et al. 2005; Howarth et al. 2003 ; Bonner et al. 2005; Heeg et al. 2008).

\section{Fig. 2}

Biosynthesis of cysteine in Arabidopsis cells. The most abundant metabolite in every cellular compartment is indicated with large bold letters. Minor OASTL-like and SATs with no clear function are also included with a question mark

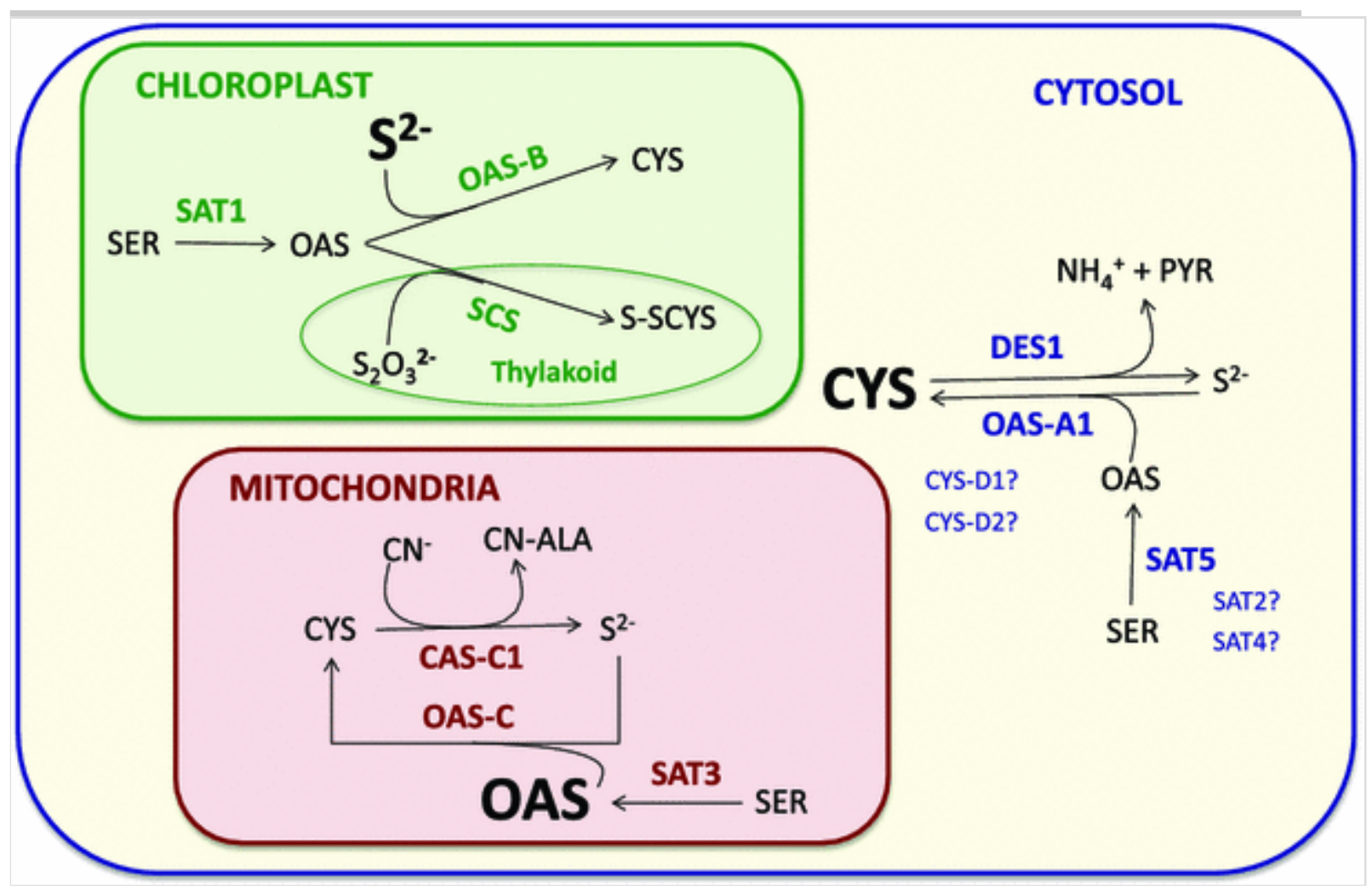

Meanwhile, the OASTL family from $A$. thaliana includes the most 
abundant cytosolic OAS-A1 (At4g14880), the plastidial OAS-B (At2g43750), and the mitochondrial OAS-C (At3g59760) isoforms (Fig. 2). These proteins are OASTLs by itself since catalyze the synthesis of Cys and are able to interact with their SAT partners (Bonner et al. 2005; Heeg et al. 2008). Other enzyme also included in the OASTL family due to the very high conservation of the encoding gene is the highly expressed isoform located in the mitochondria CAS-C1 (former CYS-C1, At3g61440), which is a $\beta$-cyanoalanine synthase, catalyzing the conversion of Cys and cyanide to hydrogen sulfide and $\beta$-cyanoalanine (Hatzfeld et al. 2000b; Yamaguchi et al. 2000). It has been demonstrated that CAS-C1 plays an essential role in the mitochondria for the maintenance of certain levels of cyanide for signaling in different plant processes (Garcia et al. 2014). Thereby, non-toxic level of cyanide in the plant regulates the root hair development and the plant immune responses (Garcia et al. 2010, 2013 ). Moreover, because hydrogen sulfide is generated by the CAS-C1 activity and is used as substrate by OAS-C, thus resulting in a cyclic pathway in the mitochondria (Fig. 2), the OAS-C is acting together with CAS-C1 in modulating the cyanide levels (Alvarez et al. 2012c).

The Arabidopsis chloroplasts contain, in addition to OAS-B, other isoform initially identified by sequence homology and which is expressed to very low levels, the protein SCS (former CS26, At3g03630) (Fig. 2). SCS is a $S$-sulfocysteine synthase, catalyzing the incorporation of thiosulfate (instead of sulfide like an authentic OASTL) to OAS to form $S$-sulfocysteine, and despite to be a minor protein, seems to play an essential role in chloroplast redox control, in contrast to the abundant OAS-B protein (Bermudez et al. 2010; Gotor et al. 2010). Interestingly, these two enzymes have other important different feature, their localization inside the chloroplast. OAS-B is located in the stroma and SCS is located in the thylakoid lumen (Bermudez et al. 2012). It is there where SCS appears to exert its essential function to proper photosynthetic performance under long-day growth conditions (Bermudez et al. 2012). Thus, it is suggested that this function could be to act as a protein sensor in the thylakoid lumen of the chloroplast redox status, in the way that if a ROS production occurs, it may originate thiosulfate due to interference with reduction of sulfite, and SCS would use the thiosulfate to produce $S$-sulfocysteine that would trigger protection mechanisms (Gotor and Romero 2013). 
Finally, in the Arabidopsis cytosol there are three remaining OASTL-like expressed in lower levels than the major OASTL-A1 (Fig. 2), the CYS-D1 (At3g04940) and CYS-D2 (At5g28020), both two poorly studied, and DES1 (At5g28030), focus of intense investigation in recent years, which has shown the enzymatic reaction it catalyzes and its specific roles in plant. DES1 is a novel L-cysteine desulfhydrase that catalyzes the desulfuration of Cys, instead of the synthesis of Cys like the OASTL, to produce sulfide plus ammonia and pyruvate (Alvarez et al. 2010).

Therefore, DES1 acts in the opposite orientation to OAS-A1, and therefore it has been proposed that both enzymes are coordinately involved in regulating the homeostasis of Cys and the generation of sulfide in the cytosol for signaling purposes (Alvarez et al. 2010, 2012b; Gotor et al. 2010, 2013; Romero et al. 2013), as described below.

\section{Cysteine homeostasis in the cytosol}

In Arabidopsis, the analysis of specific mutants of members of the SAT and OASTL family has demonstrated that mitochondria are the predominant source of OAS in vivo and that cytosol is the compartment where the major bulk of Cys synthesis takes place, while chloroplasts provide the sulfide via the assimilatory sulfate reduction (Haas et al. 2008; Heeg et al. 2008; Krueger et al. 2009; Watanabe et al. 2008a, b). However, when a compartment is affected by mutations in any SAT or OASTL isoform, the other compartments are able to provide Cys for plant viability.

The observation of the cytosol as the major site of Cys synthesis correlates well with the estimated Cys concentrations $>300$ and $<10 \mu \mathrm{M}$ in other cell compartments (Krueger et al. 2009). However, Cys is a toxic molecule when it is present above a certain concentration threshold, due to the high reactivity of its thiol. These groups are easily oxidized to form reactive sulfur species (RSS) that inhibit enzyme activities, and also generate reactive oxygen species (ROS) through the Fenton reaction catalyzed by iron and provoke an important oxidative damage (Jacob et al. 2003; Park and Imlay 2003). Consequently, Cys homeostasis must be maintained very accurately and this has been suggested to occur through the coordinated action of the OAS-A1 (Cys synthesis) and DES1 (Cys degradation) enzyme activities. This suggestion arises from the detailed study of the 
oas-al and des 1 null mutants that show opposite features. A significant reduction of the total intracellular Cys concentration (oas-al mutants) originates an imbalance between the generation and removal of ROS and accordingly the oas-al mutant plants are oxidatively stressed (LopezMartin et al. 2008a); whilst a significant increase of the Cys content (des 1 mutants) enhances the antioxidant defenses and therefore decreases the ROS production (Alvarez et al. 2010). These findings clearly suggest that the level of cysteine is a determinant of the oxidative status of the cytosol in Arabidopsis (Lopez-Martin et al. 2008b).

Thereby, a change in the Cys homeostasis in the cytosol should affect redox-signaled processes in the plant and this is precisely what is observed in both oas-al and des 1 mutants. Thus, the des 1 mutants show an early leaf senescence that is evidenced at cellular level by the accumulation of senescence-associated vacuoles (SAVs) and at the transcriptional level by the induced expression of senescence-associated genes. Conversely, the oas-al mutants exhibit neither the presence of SAVs nor induced transcripts (Alvarez et al. 2010, 2012b; Lopez-Martin et al. 2008a). Likewise it occurs in the plant responses to adverse conditions, where opposing phenotypes are observed. Under abiotic stress like Cd stress, the des 1 mutants show increased tolerance and the oas-al mutants show increased sensitivity when compared to wild type plants (Alvarez et al. 2010; Lopez-Martin et al. 2008a). Upon pathogen attack, again the same behavior, that is, the enhanced resistance to pathogens by the des 1 mutants that resemble the constitutive systemic acquired resistance mutants, and the decreased resistance to pathogens by the oas-al mutants (Alvarez et al. 2012a). Therefore, the levels of Cys in the cytosol strongly affect the plant responses to both te abiotic and biotic stress conditions, resulting evident that an increased level (to a certain threshold) induces stress tolerance/resistance. These findings also confirm the involvement of the DES1 and OAS-A1 proteins in a coordinate way to regulate the Cys homeostasis in the cytosol. All these data suggest that the accurate regulation of their activities and therefore the modulation of the Cys homeostasis in the cytosol should be critical for plant performance.

\section{Regulation of autophagy by sulfide generated from cysteine in the cytosol}

One of the essential processes for plant performance is the autophagy, 
which is a universal mechanism present in eukaryotic cells involved in the digestion of cellular content for subsequent recycling of nutrients, as well as the degradation of damaged or toxic components. It is a process that occurs constitutively, that is, occurs at basal level in growing cells, allowing recycling proteins and organelles. But also, plants induce autophagy under stress conditions such as nutrient limitation, the presence of drugs and other abiotic stresses. Besides autophagy is involved in various physiological aspects as plant development, immune response or senescence. There are three types of autophagy: microautophagy, macroautophagy and chaperone mediated autophagy (CMA). During microautophagy, the material is directly engulfed by the vacuole via tonoplast invagination releasing in the vacuolar lumen a vesicle containing cytoplasmic constituents that are further digested by the hydrolases inside the vacuole. By contrast, macroautophagy starts in the cytoplasm, where cytoplasmic constituents and organelles are sequestered in a structure of double membrane called the autophagosome. The outer membrane fuses with the tonoplast, releasing the remaining autophagic body surrounded by the inner membrane. Vacuolar hydrolases degrade the autophagic body and the degradation products are transported back to the cytosol. CMA consists in the selective degradation of cytosolic proteins by using a cytosolic chaperone HSC70, which recognizes substrate proteins containing the motif KFERQ. However, it is unclear whether CMA is present in plants (Bassham 2007; Li and Vierstra 2012; Yoshimoto 2012; Perez-Perez et al. 2012; Xiong et al. 2007; Thompson and Vierstra 2005).

The $A T G$ genes are essential for the formation of autophagosome and, first identified in yeast, are highly conserved in eukaryotes and have contributed significantly to the investigation of autophagy in these organisms. The vast majority of homologs of the yeast $A T G$ genes have been identified primarily in Arabidopsis and other plants. Most of the essential components are conserved, suggesting that the molecular basis of the core autophagy machinery is essentially the same in plants and yeast. Essential genes for the formation of autophagosome are ATG1-10, 12-14, 16-18 and 31, and the encoding proteins can be divided into five functional groups: ATG1 kinase complex, PI3 kinase complex, the ATG9 complex and the two ubiquitination-like conjugation systems ATG7-ATG10 and ATG7-ATG3 (Thompson and Vierstra 2005; Bassham et al. 2006; Yoshimoto et al. 2010). The best-studied proteins involved in 
plant autophagy are those of the ubiquitin-like conjugation systems. The first contributes to the formation of a covalently linked conjugate of ATG5 and ATG12, and the second is more unusual because the ATG8 protein is conjugated to a lipid, phosphatidylethanolamine (PE). Both conjugates are involved in the biogenesis of the autophagosome membranes, but only ATG8-PE is present upon completion of the autophagosome. Therefore, the ATG8 accumulation and lipidation have been extensively used to monitor autophagic activity in plants (Yoshimoto et al. 2004; Thompson et al. 2005; Phillips et al. 2008; Chung et al. 2009).

Precisely, the accumulation and lipidation of ATG8 isoforms in Arabidopsis promoted by the deficiency of the DES1 enzyme have allowed to interlink the Cys metabolism in the cytosol with the process of autophagy. Detailed characterization of the des 1 null mutants has enabled to get information about the role of sulfide metabolically generated from Cys in the cytosol as a signaling molecule that regulates autophagy (Alvarez et al. 2012b; Gotor et al. 2013). As described above for other plant processes, also the oas-al and des 1 mutants show opposing phenotypes related to the progression of autophagy. The absence of functional DES1 in the cytosol causes an accumulation of ATG8 proteins, predominantly for the lipidated ATG8 forms. By contrast, the same patterns of ATG8 lipidation in wild type and the oas-al mutant plants are observed (Alvarez et al. 2012b). These results may suggest that either the level of Cys in the cytosol regulates the progression of autophagy (less Cys like in the oas-al mutant plants inhibits autophagy/more Cys like in the des 1 mutant plants represses autophagy), or that specifically the absence of the DES1 enzyme activity in the cytosol promotes the induction of autophagy. Different clues coming from emerging experimental evidence that reveal sulfide to be a signaling molecule of equal importance to NO and $\mathrm{H}_{2} \mathrm{O}_{2}$ in plants, and conducting further research, allowed us to believe that the second conclusion is more plausible (Gotor et al. 2013; Romero et al. 2013).

Although hydrogen sulfide has been known for a long time as a toxic molecule for life, in recent years, it has been recognized as an important signaling molecule both in animal and plant systems (Wang 2012; Garcia-Mata and Lamattina 2013; Lisjak et al. 2013). In plants, numerous studies have shown that sulfide increases tolerance/resistance against stress 
conditions, such as metal stress (Zhang et al. 2008; Dawood et al. 2012; Wang et al. 2010; Sun et al. 2013; Li et al. 2012), drought (Jin et al. 2011 ), hypoxia (Cheng et al. 2013), salinity (Christou et al. 2013; Wang et al. 2012), and heat ( $\mathrm{Li}$ et al. 2013). Moreover, sulfide has been also demonstrated to regulate many essential plant processes, like photosynthesis (Chen et al. 2011), stomatal movement (Garcia-Mata and Lamattina 2010; Lisjak et al. 2010), flower senescence (Zhang et al. 2011), and postharvest life of fruits (Hu et al. 2012). Therefore, because DES1 catalyzes the enzymatic desulfuration of Cys to sulfide, the DES1 mutation provokes a reduction in the capacity of the cytosol to release sulfide. Consequently, restoring the capacity of sulfide generation, either by genetic complementation or by exogenous applied sulfide, is able to rescue the induction of autophagy observed in the des 1 null mutants (Fig. 3). When the des 1 mutant is complemented with the full-length DES 1 cDNA fragment, the same pattern of ATG8 accumulation as the wild type is observed (Fig. 3), showing also the same endogenous measured $\mathrm{H}_{2} \mathrm{~S}$ concentrations (Alvarez et al. 2012b). Analogously, the exogenous addition of sulfide rescues the autophagy activation phenotype of the des 1 mutant, as observed in the immunoblot analysis where a significant reduction of ATG8 accumulation and lipidation is detected (Fig. 3) (Alvarez et al. 2012b). All these data reinforce the idea that is the intracellular sulfide generated from Cys in the cytosol that acts regulating the process of autophagy.

Fig. 3 
Effect of genetic complementation and exogenous sulfide on the induced autophagy of the des 1 null mutant. A. thaliana wild type, des 1 mutant and the complemented-des 1 line plants were grown in soil for 4 weeks under physiological conditions, and the NaHS-treated des 1 mutant plants were grown for 20 days under physiological conditions and 10 additional days irrigated with $200 \mu \mathrm{M}$ NaHS. Protein extracts were prepared from leaves as described (Alvarez et al. 2012b). For immunoblot analysis, $60 \mu \mathrm{g}$ of protein extracts were resolved by $15 \%$ SDS-PAGE and transfer to membrane that were incubated with anti-Cr-ATG8 primary (Perez-Perez et al. 2010) and horseradish peroxidase-conjugated anti-rabbit secondary antibodies diluted 1:2,000 and 1:10,000, respectively. The previous Ponceau staining of the membrane is shown as the protein loading control. More details in (Alvarez et al. 2012b) (http://www.plantcell.org; copyright American Society of Plant Biologists)

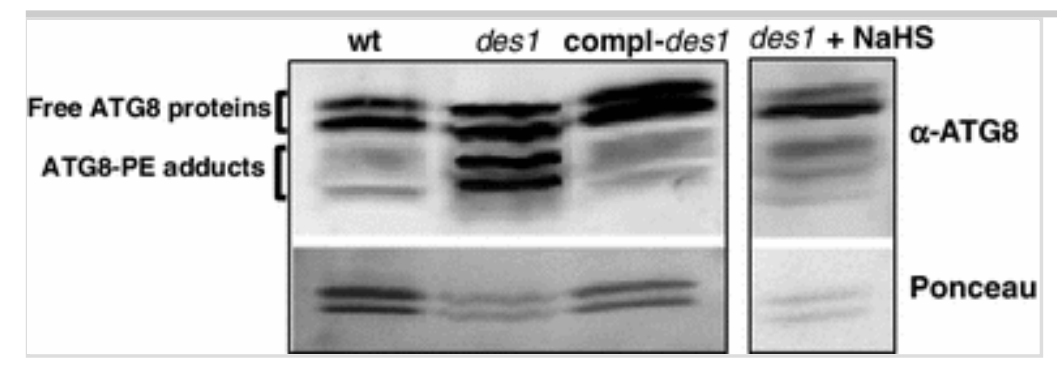

Different studies have shown an induction of autophagy under nutrient starvation conditions in plants (Rose et al. 2006; Thompson et al. 2005; Yoshimoto et al. 2004; Chung et al. 2009; Contento et al. 2004; Xiong et al. 2005 ). Interestingly, exogenous sulfide also suppresses the induction of autophagy in wild type plants when are subjected to carbon starvation (Alvarez et al. 2012b). Thus, it seems that sulfide specifically generated from the degradation of Cys inside the cytosol, acts as a signaling molecule and regulates negatively the autophagy induced by different situations. This regulation has shown independent of its role as nutrient as it has been demonstrated in the studied plant system that does not exhibit sulfur limitation (Alvarez et al. 2012b).

In addition to the published in plant systems, it was recently reported in yeast that, although nitrogen deficit is also a common trigger of autophagy, some yeast cells induce autophagy upon switch from a rich to minimal media without nitrogen starvation and that sulfur-containing amino acids, 
cysteine and methionine, inhibit such non-nitrogen-starvation-induced autophagy (Sutter et al. 2013). The mechanism underlying this regulatory system of autophagy in yeast indicates that the level of cysteine and methionine and its downstream metabolite $\mathrm{S}$-adenosylmethionine regulates the methylation status of the protein phosphatase PP2A which in turn promotes dephosphorylation of Npr2p, a component of a conserved complex that regulates autophagy and other growth-related processes (Laxman et al. 2014; Sutter et al. 2013). The data shown in plant and yeast indicate that sulfur-amino acids act as sensors of the metabolic status and trigger signaling mechanisms that control important cellular processes.

\section{Conclusions and future perspectives}

The amino acid cysteine is an essential metabolite for plant development. It is the product of the photosynthetic sulfate assimilation pathway and therefore the entry point of the reduced sulfur into the metabolism firstly of plants but also for humans. Most remarkable, Cys is essential for protein structure, function and regulation, and equally important Cys is the precursor of a variety of sulfur-containing metabolites essential for plant life. In recent years, an intense investigation on the enzymes involved in the biosynthesis of cysteine, mainly in A. thaliana, has been developed, and a very significant knowledge has emerged. One important breakthrough has been the demonstration that cytosol is the compartment where Cys biosynthesis mainly takes place, which has highlighted the relevance of the homeostasis of Cys in the cytosol. Recent findings has shown that alteration of this homeostasis strongly affects the plant responses to stress conditions and that its modulation by the coordinate action of the enzymes OAS-A1 (catalyzing the Cys synthesis) and DES1 (catalyzing the desulfuration of Cys to sulfide) should be critical for plant performance (Fig. 4). In addition, another relevant breakthrough has been the implication of Cys in the generation of the molecule of sulfide in the plant cytosol for signaling purposes. Thus, it has been concluded that the cytosolic DES1 enzyme is the responsible for modulating the generation of sulfide that acts as a signaling molecule regulating an essential process for any eukaryotic organism like the autophagy. This conclusion opens many areas of potential investigation; such as to determine the mechanisms of $\mathrm{H}_{2} \mathrm{~S}$ action and its molecular targets related to autophagy or any other plant processes. Furthermore, the mechanism underlying the modulation of 
sulfide generation by the DES1 enzyme, the specific function and coordination of DES 1 and the OAS-A 1 in the signaling of different plant processes, the regulation of these enzymes, and so on.

Fig. 4

Modulation of cysteine/sulfide levels in the cytosol. Different situations affect the levels of cysteine and sulfide in the cytosol through the coordination of the enzymatic reactions catalyzed by OAS-A1 and DES1, what is critical for adequate plant performance

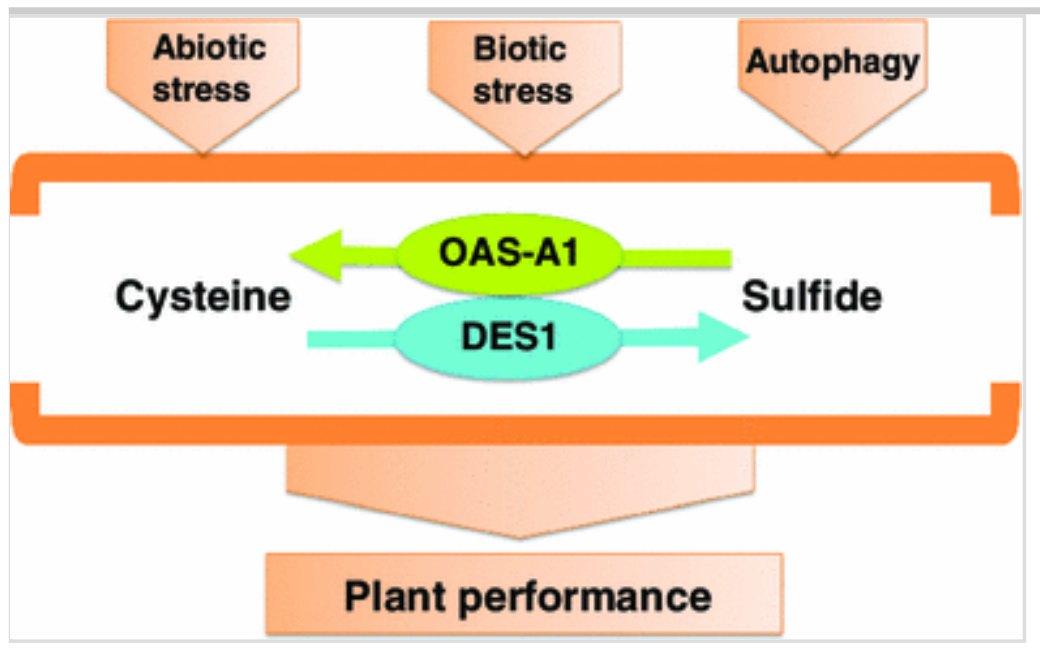

\title{
Acknowledgments
}

This work was funded in part by the European Regional Development Fund through the Ministerio de Economia y Competitividad (grants no. BIO2010-15201and BIO2013-44648) and the Junta de Andalucía (grant no. CVI-7190). A.M.L-M. thanks the Ministerio de Economia y Competitividad for fellowship support through the program of Formación de Personal Investigador. A.A. thanks the Consejo Superior de Investigaciones Científicas for economic support provided by the postdoctoral program of the Junta de Ampliación de Estudios part-financed by the European Social Fund.

\section{Conflict of interest}

The authors declare that they have no conflict of interest.

\section{References}

\author{
Alvarez C, Calo L, Romero LC, Garcia I, Gotor C (2010) An
}


$O$-acetylserine(thiol)lyase homolog with L-cysteine desulfhydrase activity regulates cysteine homeostasis in Arabidopsis. Plant Physiol 152(2):656-669. doi:10.1104/pp.109.147975

Alvarez C, Bermudez MA, Romero LC, Gotor C, Garcia I (2012a) Cysteine homeostasis plays an essential role in plant immunity. New Phytol 193(1):165-177. doi:10.1111/j.1469-8137.2011.03889.x

Alvarez C, Garcia I, Moreno I, Perez-Perez ME, Crespo JL, Romero LC, Gotor C (2012b) Cysteine-generated sulfide in the cytosol negatively regulates autophagy and modulates the transcriptional profile in Arabidopsis. Plant Cell 24(11):4621-4634.

doi:10.1105/tpc.112.105403

Alvarez C, Garcia I, Romero LC, Gotor C (2012c) Mitochondrial sulfide detoxification requires a functional isoform $O$-acetylserine(thiol)lyase C in Arabidopsis thaliana. Mol Plant 5(6):1217-1226. doi:10.1093/mp/sss043

Bassham DC (2007) Plant autophagy_more than a starvation response. Curr Opin Plant Biol 10(6):587-593. doi:10.1016/j.pbi.2007.06.006

Bassham DC, Laporte M, Marty F, Moriyasu Y, Ohsumi Y, Olsen LJ, Yoshimoto K (2006) Autophagy in development and stress responses of plants. Autophagy 2(1):2-11 2092 [pii]

Bermudez MA, Paez-Ochoa MA, Gotor C, Romero LC (2010) Arabidopsis $S$-sulfocysteine synthase activity is essential for chloroplast function and long-day light-dependent redox control. Plant Cell 22(2):403-416. doi:10.1105/tpc.109.071985

Bermudez MA, Galmes J, Moreno I, Mullineaux PM, Gotor C, Romero LC (2012) Photosynthetic adaptation to length of day is dependent on s-sulfocysteine synthase activity in the thylakoid lumen. Plant Physiol 160(1):274-288. doi:10.1104/pp.112.201491

Bleecker AB, Kende H (2000) Ethylene: a gaseous signal molecule in plants. Annu Rev Cell Dev Biol 16:1-18.

doi:10.1146/annurev.cellbio.16.1.1 
Bonner ER, Cahoon RE, Knapke SM, Jez JM (2005) Molecular basis of cysteine biosynthesis in plants: structural and functional analysis of O-acetylserine sulfhydrylase from Arabidopsis thaliana. J Biol Chem 280(46):38803-38813

Buchanan BB, Balmer Y (2005) Redox regulation: a broadening horizon. Annu Rev Plant Biol 56:187-220

Buchner P, Stuiver CE, Westerman S, Wirtz M, Hell R, Hawkesford MJ, De Kok LJ (2004) Regulation of sulfate uptake and expression of sulfate transporter genes in Brassica oleracea as affected by atmospheric $\mathrm{H}(2) \mathrm{S}$ and pedospheric sulfate nutrition. Plant Physiol 136(2):3396-3408

Chen J, Wu FH, Wang WH, Zheng CJ, Lin GH, Dong XJ, He JX, Pei ZM, Zheng HL (2011) Hydrogen sulphide enhances photosynthesis through promoting chloroplast biogenesis, photosynthetic enzyme expression, and thiol redox modification in Spinacia oleracea seedlings. J Exp Bot 62(13):4481-4493. doi:10.1093/jxb/err145

Cheng W, Zhang L, Jiao C, Su M, Yang T, Zhou L, Peng R, Wang R, Wang C (2013) Hydrogen sulfide alleviates hypoxia-induced root tip death in Pisum sativum. Plant Physiol Biochem 70:278-286.

doi:10.1016/j.plaphy.2013.05.042

Christou A, Manganaris GA, Papadopoulos I, Fotopoulos V (2013) Hydrogen sulfide induces systemic tolerance to salinity and non-ionic osmotic stress in strawberry plants through modification of reactive species biosynthesis and transcriptional regulation of multiple defence pathways. J Exp Bot 64(7):1953-1966. doi:10.1093/jxb/ert055

Chung T, Suttangkakul A, Vierstra RD (2009) The ATG autophagic conjugation system in maize: aTG transcripts and abundance of the ATG8-lipid adduct are regulated by development and nutrient availability. Plant Physiol 149(1):220-234. doi:10.1104/pp.108.126714

Contento AL, Kim SJ, Bassham DC (2004) Transcriptome profiling of the response of Arabidopsis suspension culture cells to Suc starvation. Plant Physiol 135(4):2330-2347. doi:10.1104/pp.104.044362 
Dawood M, Cao F, Jahangir MM, Zhang G, Wu F (2012) Alleviation of aluminum toxicity by hydrogen sulfide is related to elevated ATPase, and suppressed aluminum uptake and oxidative stress in barley. $\mathrm{J}$ Hazard Mater 209-210:121-128. doi:10.1016/j.jhazmat.2011.12.076

Dixon DP, Lapthorn A, Edwards R (2002) Plant glutathione transferases. Genome Biol 3(3):REVIEWS3004

Droux M (2004) Sulfur assimilation and the role of sulfur in plant metabolism: a survey. Photosynth Res 79(3):331-348

Droux M, Ruffet ML, Douce R, Job D (1998) Interactions between serine acetyltransferase and $O$-acetylserine(thiol)lyase in higher plants - structural and kinetic properties of the free and bound enzymes. Eur $\mathrm{J}$ Biochem 255(1):235-245

Foyer $\mathrm{CH}$, Noctor $\mathrm{G}$ (2011) Ascorbate and glutathione: the heart of the redox hub. Plant Physiol 155(1):2-18. doi:10.1104/pp.110.167569

Francois JA, Kumaran S, Jez JM (2006) Structural basis for interaction of $O$-acetylserine sulfhydrylase and serine acetyltransferase in the Arabidopsis cysteine synthase complex. Plant Cell 18(12):3647-3655

Garcia I, Castellano JM, Vioque B, Solano R, Gotor C, Romero LC (2010) Mitochondrial \{beta\}-cyanoalanine synthase is essential for root hair formation in Arabidopsis thaliana. Plant Cell 22(10):3268-3279. doi: $10.1105 /$ tpc. 110.076828

Garcia I, Rosas T, Bejarano ER, Gotor C, Romero LC (2013) Transient transcriptional regulation of the CYS-C1 gene and cyanide accumulation upon pathogen infection in the plant immune response. Plant Physiol 162(4):2015-2027. doi:10.1104/pp.113.219436

Garcia I, Gotor C, Romero LC (2014) Beyond toxicity: a regulatory role for mitochondrial cyanide. Plant Signal Behav 8(12) AQ2

Garcia-Mata C, Lamattina L (2010) Hydrogen sulphide, a novel gasotransmitter involved in guard cell signalling. New Phytol 
188(4):977-984. doi:10.1111/j.1469-8137.2010.03465.x

Garcia-Mata C, Lamattina L (2013) Gasotransmitters are emerging as new guard cell signaling molecules and regulators of leaf gas exchange. Plant Sci 201-202:66-73. doi:10.1016/j.plantsci.2012.11.007

Gotor C, Romero LC (2013) S-sulfocysteine synthase function in sensing chloroplast redox status. Plant Signal Behav 8(3)

Gotor C, Alvarez C, Bermudez MA, Moreno I, Garcia I, Romero LC (2010) Low abundance does not mean less importance in cysteine metabolism. Plant Signal Behav 5(8)

Gotor C, Garcia I, Crespo JL, Romero LC (2013) Sulfide as a signaling molecule in autophagy. Autophagy 9(4):609-611.

doi:10.4161/auto.23460

Gutierrez-Marcos JF, Roberts MA, Campbell EI, Wray JL (1996) Three members of a novel small gene-family from Arabidopsis thaliana able to complement functionally an Escherichia coli mutant defective in PAPS reductase activity encode proteins with a thioredoxin-like domain and "APS reductase" activity. Proc Natl Acad Sci USA 93(23):13377-13382

Haag AF, Kerscher B, Dall'Angelo S, Sani M, Longhi R, Baloban M, Wilson HM, Mergaert P, Zanda M, Ferguson GP (2012) Role of cysteine residues and disulfide bonds in the activity of a legume root nodule-specific, cysteine-rich peptide. J Biol Chem 287(14):10791-10798. doi:10.1074/jbc.M111.311316

Haas FH, Heeg C, Queiroz R, Bauer A, Wirtz M, Hell R (2008) Mitochondrial serine acetyltransferase functions as a pacemaker of cysteine synthesis in plant cells. Plant Physiol 148(2):1055-1067

Hatzfeld Y, Lee S, Lee M, Leustek T, Saito K (2000a) Functional characterization of a gene encoding a fourth ATP sulfurylase isoform from Arabidopsis thaliana. Gene 248(1-2):51-58

Hatzfeld Y, Maruyama A, Schmidt A, Noji M, Ishizawa K, Saito K 
(2000b) Beta-cyanoalanine synthase is a mitochondrial cysteine synthase-like protein in spinach and Arabidopsis. Plant Physiol 123(3):1163-1171

Heeg C, Kruse C, Jost R, Gutensohn M, Ruppert T, Wirtz M, Hell R (2008) Analysis of the Arabidopsis $O$-acetylserine(thiol)lyase gene family demonstrates compartment-specific differences in the regulation of cysteine synthesis. Plant Cell 20(1):168-185

Howarth JR, Dominguez-Solis JR, Gutierrez-Alcala G, Wray JL, Romero LC, Gotor C (2003) The serine acetyltransferase gene family in Arabidopsis thaliana and the regulation of its expression by cadmium. Plant Mol Biol 51(4):589-598

Hu LY, Hu SL, Wu J, Li YH, Zheng JL, Wei ZJ, Liu J, Wang HL, Liu YS, Zhang H (2012) Hydrogen sulfide prolongs postharvest shelf life of strawberry and plays an antioxidative role in fruits. J Agric Food Chem 60(35):8684-8693. doi:10.1021/jf300728h

Jacob C, Giles GI, Giles NM, Sies H (2003) Sulfur and selenium: the role of oxidation state in protein structure and function. Angew Chem Int Edn Engl 42(39):4742-4758

Jez JM, Dey S (2013) The cysteine regulatory complex from plants and microbes: what was old is new again. Curr Opin Struct Biol 23(2):302-310. doi:10.1016/j.sbi.2013.02.011

Jin Z, Shen J, Qiao Z, Yang G, Wang R, Pei Y (2011) Hydrogen sulfide improves drought resistance in Arabidopsis thaliana. Biochem Biophys Res Commun 414(3):481-486. doi:10.1016/j.bbrc.2011.09.090

Kawashima CG, Berkowitz O, Hell R, Noji M, Saito K (2005) Characterization and expression analysis of a serine acetyltransferase gene family involved in a key step of the sulfur assimilation pathway in Arabidopsis. Plant Physiol 137(1):220-230

Khan MS, Haas FH, Samami AA, Gholami AM, Bauer A, Fellenberg K, Reichelt M, Hansch R, Mendel RR, Meyer AJ, Wirtz M, Hell R (2010) Sulfite reductase defines a newly discovered bottleneck for assimilatory 
sulfate reduction and is essential for growth and development in Arabidopsis thaliana. Plant Cell 22(4):1216-1231.

doi:10.1105/tpc. 110.074088

Kopriva S, Koprivova A (2004) Plant adenosine 5'-phosphosulphate reductase: the past, the present, and the future. J Exp Bot 55(404):1775-1783. doi:10.1093/jxb/erh185

Krueger S, Niehl A, Lopez Martin MC, Steinhauser D, Donath A, Hildebrandt T, Romero LC, Hoefgen R, Gotor C, Hesse H (2009) Analysis of cytosolic and plastidic serine acetyltransferase mutants and subcellular metabolite distributions suggests interplay of the cellular compartments for cysteine biosynthesis in Arabidopsis. Plant Cell Environ 32(4):349-367. doi:10.1111/j.1365-3040.2008.01928.x

Laxman S, Sutter BM, Tu BP (2014) Methionine is a signal of amino acid sufficiency that inhibits autophagy through the methylation of PP2A. Autophagy 10(2):386-387. doi:10.4161/auto.27485

Li F, Vierstra RD (2012) Autophagy: a multifaceted intracellular system for bulk and selective recycling. Trends Plant Sci 17(9):526-537. doi:10.1016/j.tplants.2012.05.006

Li L, Wang Y, Shen W (2012) Roles of hydrogen sulfide and nitric oxide in the alleviation of cadmium-induced oxidative damage in alfalfa seedling roots. Biometals 25(3):617-631.

doi:10.1007/s 10534-012-9551-9

Li ZG, Yang SZ, Long WB, Yang GX, Shen ZZ (2013) Hydrogen sulphide may be a novel downstream signal molecule in nitric oxideinduced heat tolerance of maize (Zea mays L.) seedlings. Plant Cell Environ 36(8):1564-1572. doi:10.1111/pce.12092

Lisjak M, Srivastava N, Teklic T, Civale L, Lewandowski K, Wilson I, Wood ME, Whiteman M, Hancock JT (2010) A novel hydrogen sulfide donor causes stomatal opening and reduces nitric oxide accumulation. Plant Physiol Biochem 48(12):931-935.

doi:10.1016/j.plaphy.2010.09.016 
Lisjak M, Teklic T, Wilson ID, Whiteman M, Hancock JT (2013) Hydrogen sulfide: environmental factor or signalling molecule? Plant Cell Environ 36(9):1607-1616. doi:10.1111/pce.12073

Lopez-Martin MC, Becana M, Romero LC, Gotor C (2008a) Knocking out cytosolic cysteine synthesis compromises the antioxidant capacity of the cytosol to maintain discrete concentrations of hydrogen peroxide in Arabidopsis. Plant Physiol 147(2):562-572

Lopez-Martin MC, Romero LC, Gotor C (2008b) Cytosolic cysteine in redox signaling. Plant Signal Behav 3(10):880-881

Mendoza-Cozatl DG, Jobe TO, Hauser F, Schroeder JI (2011)

Long-distance transport, vacuolar sequestration, tolerance, and transcriptional responses induced by cadmium and arsenic. Curr Opin Plant Biol 14(5):554-562. doi:10.1016/j.pbi.2011.07.004

Noctor G, Mhamdi A, Chaouch S, Han Y, Neukermans J, MarquezGarcia B, Queval G, Foyer CH (2012) Glutathione in plants: an integrated overview. Plant Cell Environ 35(2):454-484.

doi:10.1111/j.1365-3040.2011.02400.x

Park S, Imlay JA (2003) High levels of intracellular cysteine promote oxidative DNA damage by driving the fenton reaction. J Bacteriol 185(6):1942-1950

Perez-Perez ME, Florencio FJ, Crespo JL (2010) Inhibition of target of rapamycin signaling and stress activate autophagy in Chlamydomonas reinhardtii. Plant Physiol 152(4):1874-1888.

doi:10.1104/pp.109.152520

Perez-Perez ME, Lemaire SD, Crespo JL (2012) Reactive oxygen species and autophagy in plants and algae. Plant Physiol 160(1):156-164. doi:10.1104/pp.112.199992

Phillips AR, Suttangkakul A, Vierstra RD (2008) The ATG12conjugating enzyme ATG10 is essential for autophagic vesicle formation in Arabidopsis thaliana. Genetics 178(3):1339-1353. doi:10.1534/genetics.107.086199 
Rausch T, Wachter A (2005) Sulfur metabolism: a versatile platform for launching defence operations. Trends Plant Sci 10(10):503-509

Rea PA (2012) Phytochelatin synthase: of a protease a peptide polymerase made. Physiol Plant 145(1):154-164.

doi:10.1111/j.1399-3054.2012.01571.x

Richau KH, Kaschani F, Verdoes M, Pansuriya TC, Niessen S, Stuber K, Colby T, Overkleeft HS, Bogyo M, Van der Hoorn RA (2012) Subclassification and biochemical analysis of plant papain-like cysteine proteases displays subfamily-specific characteristics. Plant Physiol 158(4):1583-1599. doi:10.1104/pp.112.194001

Romero LC, Garcia I, Gotor C (2013) L-Cysteine desulfhydrase 1 modulates the generation of the signaling molecule sulfide in plant cytosol. Plant Signal Behav 8(5)

Romero LC, Aroca MA, Laureano-Marin AM, Moreno I, Garcia I, Gotor C (2014) Cysteine and cysteine-related signaling pathways in Arabidopsis thaliana. Mol Plant 7(2):264-276. doi:10.1093/mp/sst168

Rose TL, Bonneau L, Der C, Marty-Mazars D, Marty F (2006) Starvation-induced expression of autophagy-related genes in Arabidopsis. Biol Cell 98(1):53-67. doi:10.1042/BC20040516

Setya A, Murillo M, Leustek T (1996) Sulfate reduction in higher plants: molecular evidence for a novel 5'-adenylylsulfate reductase. Proc Natl Acad Sci USA 93(23):13383-13388

Sun J, Wang R, Zhang X, Yu Y, Zhao R, Li Z, Chen S (2013) Hydrogen sulfide alleviates cadmium toxicity through regulations of cadmium transport across the plasma and vacuolar membranes in Populus euphratica cells. Plant Physiol Biochem 65:67-74. doi:10.1016/j.plaphy.2013.01.003

Sutter BM, Wu X, Laxman S, Tu BP (2013) Methionine inhibits autophagy and promotes growth by inducing the SAM-responsive methylation of PP2A. Cell 154(2):403-415. doi:10.1016/j.cell.2013.06.041 
Takahashi H (2010) Regulation of sulfate transport and assimilation in plants, Chapter 4. In: Kwang WJ (ed) International review of cell and molecular biology, vol 281. Academic Press, New York, pp 129-159. doi:10.1016/S1937-6448(10)81004-4

Takahashi H, Kopriva S, Giordano M, Saito K, Hell R (2011) Sulfur assimilation in photosynthetic organisms: molecular functions and regulations of transporters and assimilatory enzymes. Annu Rev Plant Biol 62:157-184. doi:10.1146/annurev-arplant-042110-103921

Thompson AR, Vierstra RD (2005) Autophagic recycling: lessons from yeast help define the process in plants. Curr Opin Plant Biol 8(2):165-173. doi:10.1016/j.pbi.2005.01.013

Thompson AR, Doelling JH, Suttangkakul A, Vierstra RD (2005) Autophagic nutrient recycling in Arabidopsis directed by the ATG8 and ATG12 conjugation pathways. Plant Physiol 138(4):2097-2110. doi:10.1104/pp.105.060673

Van Hoewyk D, Pilon M, Pilon-Smits EAH (2008) The functions of NifS-like proteins in plant sulfur and selenium metabolism. Plant Sci 174(2):117-123. doi:10.1016/j.plantsci.2007.10.004

Vauclare P, Kopriva S, Fell D, Suter M, Sticher L, von Ballmoos P, Krahenbuhl U, den Camp RO, Brunold C (2002) Flux control of sulphate assimilation in Arabidopsis thaliana: adenosine

5'-phosphosulphate reductase is more susceptible than ATP sulphurylase to negative control by thiols. Plant J 31(6):729-740

Wang R (2012) Physiological implications of hydrogen sulfide: a whiff exploration that blossomed. Physiol Rev 92(2):791-896.

doi:10.1152/physrev.00017.2011

Wang BL, Shi L, Li YX, Zhang WH (2010) Boron toxicity is alleviated by hydrogen sulfide in cucumber (Cucumis sativus L.) seedlings. Planta 231(6):1301-1309. doi:10.1007/s00425-010-1134-9

Wang Y, Li L, Cui W, Xu S, Shen W, Wang R (2012) Hydrogen sulfide enhances alfalfa (Medicago sativa) tolerance against salinity during 
seed germination by nitric oxide pathway. Plant Soil 351(1-2):107-119

Watanabe M, Kusano M, Oikawa A, Fukushima A, Noji M, Saito K (2008a) Physiological roles of the beta-substituted alanine synthase gene family in Arabidopsis. Plant Physiol 146(1):310-320

Watanabe M, Mochida K, Kato T, Tabata S, Yoshimoto N, Noji M, Saito $\mathrm{K}$ (2008b) Comparative genomics and reverse genetics analysis reveal indispensable functions of the serine acetyltransferase gene family in Arabidopsis. Plant Cell 20(9):2484-2496

Wirtz M, Droux M (2005) Synthesis of the sulfur amino acids: cysteine and methionine. Photosynth Res 86(3):345-362

Wirtz M, Hell R (2006) Functional analysis of the cysteine synthase protein complex from plants: structural, biochemical and regulatory properties. J Plant Physiol 163(3):273-286

Wirtz M, Hell R (2007) Dominant-negative modification reveals the regulatory function of the multimeric cysteine synthase protein complex in transgenic tobacco. Plant Cell 19(2):625-639

Wirtz M, Droux M, Hell R (2004) $O$-acetylserine(thiol)lyase: an enigmatic enzyme of plant cysteine biosynthesis revisited in Arabidopsis thaliana. J Exp Bot 55(404):1785-1798. doi:10.1093/jxb /erh201

Xiong Y, Contento AL, Bassham DC (2005) AtATG18a is required for the formation of autophagosomes during nutrient stress and senescence in Arabidopsis thaliana. Plant J 42(4):535-546.

doi:10.1111/j.1365-313X.2005.02397.x

Xiong Y, Contento AL, Nguyen PQ, Bassham DC (2007) Degradation of oxidized proteins by autophagy during oxidative stress in Arabidopsis. Plant Physiol 143(1):291-299. doi:10.1104/pp.106.092106

Yamaguchi Y, Nakamura T, Kusano T, Sano H (2000) Three Arabidopsis genes encoding proteins with differential activities for cysteine synthase and beta-cyanoalanine synthase. Plant Cell Physiol 
Yi H, Galant A, Ravilious GE, Preuss ML, Jez JM (2010) Sensing sulfur conditions: simple to complex protein regulatory mechanisms in plant thiol metabolism. Mol Plant 3(2):269-279. doi:10.1093/mp /ssp112

Yoshimoto K (2012) Beginning to understand autophagy, an intracellular self-degradation system in plants. Plant Cell Physiol 53(8):1355-1365. doi:10.1093/pcp/pcs099

Yoshimoto K, Hanaoka H, Sato S, Kato T, Tabata S, Noda T, Ohsumi Y (2004) Processing of ATG8s, ubiquitin-like proteins, and their deconjugation by ATG4s are essential for plant autophagy. Plant Cell 16(11):2967-2983. doi:10.1105/tpc.104.025395

Yoshimoto N, Inoue E, Watanabe-Takahashi A, Saito K, Takahashi H (2007) Posttranscriptional regulation of high-affinity sulfate transporters in Arabidopsis by sulfur nutrition. Plant Physiol 145(2):378-388. doi:10.1104/pp.107.105742

Yoshimoto K, Takano Y, Sakai Y (2010) Autophagy in plants and phytopathogens. FEBS Lett 584(7):1350-1358.

doi:10.1016/j.febslet.2010.01.007

Zhang H, Hu LY, Hu KD, He YD, Wang SH, Luo JP (2008) Hydrogen sulfide promotes wheat seed germination and alleviates oxidative damage against copper stress. J Integr Plant Biol 50(12):1518-1529. doi:10.1111/j.1744-7909.2008.00769.x

Zhang H, Hu S-L, Zhang Z-J, Hu L-Y, Jiang C-X, Wei Z-J, Liu J, Wang H-L, Jiang S-T (2011) Hydrogen sulfide acts as a regulator of flower senescence in plants. Postharvest Biol Technol 60:251-257 